\title{
Parametric amplification on rf-induced steps in a Josephson tunnel junction
}

\author{
Sørensen, O.H.; Pedersen, Niels Falsig; Mygind, Jesper; Dueholm, B.
}

Published in:

Journal of Applied Physics

Link to article, DOI:

10.1063/1.326184

Publication date:

1979

Document Version

Publisher's PDF, also known as Version of record

Link back to DTU Orbit

Citation (APA):

Sørensen, O. H., Pedersen, N. F., Mygind, J., \& Dueholm, B. (1979). Parametric amplification on rf-induced steps in a Josephson tunnel junction. Journal of Applied Physics, 50(4), 2988-2990.

https://doi.org/10.1063/1.326184

\section{General rights}

Copyright and moral rights for the publications made accessible in the public portal are retained by the authors and/or other copyright owners and it is a condition of accessing publications that users recognise and abide by the legal requirements associated with these rights.

- Users may download and print one copy of any publication from the public portal for the purpose of private study or research.

- You may not further distribute the material or use it for any profit-making activity or commercial gain

- You may freely distribute the URL identifying the publication in the public portal

If you believe that this document breaches copyright please contact us providing details, and we will remove access to the work immediately and investigate your claim. 


\title{
Parametric amplification on rf-induced steps in a Josephson tunnel junction
}

\author{
O. H. Soerensen, N. F. Pedersen, J. Mygind, and B. Dueholm
}

Physics Laboratory I, The Technical University of Denmark, DK-2800 Lyngby, Denmark

(Received 13 July 1978; accepted for publication 14 September 1978)

\begin{abstract}
Parametric effects including amplification in a singly degenerate mode have been observed in Josephson tunnel junctions at dc bias points on rf-induced steps. Net gain at $9 \mathrm{GHz}$ was achieved with a bias on the fundamental $18-\mathrm{GHz}$ step and subharmonic selfoscillations were seen on 18 and $70-\mathrm{GHz}$ rf-induced steps even at voltages approaching the energy gap. A qualitative explanation of the results is presented.
\end{abstract}

PACS numbers: $75.40 .+\mathrm{r}, 74.30 . \mathrm{Gn}, 84.30 . \mathrm{Dx}, 85.25 .+\mathrm{k}$

Josephson junctions have been operated successfully as externally pumped parametric amplifiers in two different modes: the doubly degenerate mode where the pump, signal, and idler frequencies are about equal ${ }^{1-3}$ and the singly degenerate mode where the pump frequency is about twice the signal and idler frequencies. ${ }^{4}$ In the doubly degenerate mode the junctions were unbiased, i.e., at zero dc voltage and zero dc current. In the singly degenerate mode the junctions were biased at a finite dc current but still at zero dc voltage.

Here, we report the experimental observation that parametric phenomena strongly resembling those seen under zero-voltage bias conditions may occur at bias points on the rf-induced steps in the $I$ - $V$ curve. Parametric amplification of a 9-GHz signal with a net gain of $18 \mathrm{~dB}$ has been achieved at a bias point on the fundamental $18-\mathrm{GHz}$ step. Parametric excitation of the self-oscillation at one-half the pump frequency has been observed up to and including the seventhorder 18-GHz step. The one-half harmonic parametric oscillation was also seen on the first- to fourth-order $70-\mathrm{GHz}$ steps.

The junctions were $\mathrm{Sn}-\mathrm{O}-\mathrm{Sn}$ tunnel junctions with areas from $10 \times 10$ to $100 \times 200 \mu \mathrm{m}^{2}$ and with maximum current densities (at $T=0 \mathrm{~K}$ ) from 20 to $300 \mathrm{~A} / \mathrm{cm}^{2}$. The junctions were mounted across the end of a microwave impedance transformer as previously described. ${ }^{4,5}$

The experiments were performed using a standard oneport reflection amplifier configuration with a circulator to separate the input and output signals. The $9-$ or $35-\mathrm{GHz}$ signals from the junction were detected in conventional superheterodyne receivers with an i.f. frequency of $70 \mathrm{MHz}$ and a $10-\mathrm{MHz}$ bandwidth. The overall noise figures were 10 and $6 \mathrm{~dB}$ for the $X$ band and the $K a$-band receivers, respectively. The i.f. output was displayed on a spectrum analyzer. The pump signals at 18 or $70 \mathrm{GHz}$ were applied to the junctions from the back via loosely coupled lines.

Some representative recordings are shown in Fig. 1. Here, the junction was biased on the fundamental $(n=1)$ $18-\mathrm{GHz}$ step. Trace a shows the input signal applied to the amplifier. Traces $b$ and $c$ are the reflected signals corresponding to two different bias points on the $n=1$ step of the $I-V$ curve shown in the right-hand part of Fig. 1 . The gain obtained here is 8 and $13 \mathrm{~dB}$, respectively. The detected power at the signal and idler frequencies is of the same magnitude and the self-oscillation is not excited. The smooth background curve in trace a is due to mixer and i.f.-amplifier noise and reproduces the i.f. passband. In traces $b$ and $c$ we observe a very pronounced rise in the noise output such that the signal-to-noise ratio is only just conserved (trace b) or even reduced (trace c), i.e., the overall noise temperature of the reflection amplifier in front of the superheterodyne receiver is at least comparable to that of the receiver front end. We have not yet determined the losses in the coupling circuit and, hence, the noise temperature of the Josephson element itself cannot be calculated. The amplitude and frequency distribution of the noise is seen to vary with the bias conditions. A similar behavior is often observed with Josephson-junction parametric amplifiers operated at zero dc voltage in the doubly ${ }^{6}$ as well as in the singly ${ }^{4,5}$ degenerate modes. We shall not attempt here to explain the excessive amplifier noise but

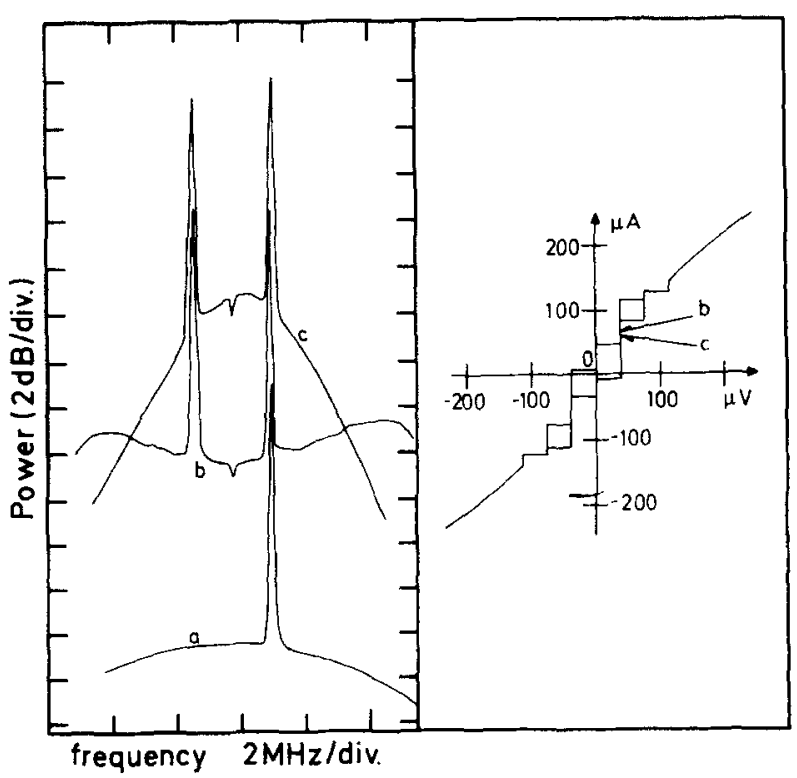

FIG. 1. Spectrum analyzer displays (center frequency: $8.7 \mathrm{GHz}$; i.f. bandwidth: $100 \mathrm{kHz}$ ). Trace a-input signal; traces $b$ and $c$-reflected signals correspondomg to bias points points shown in the right-hand part. 


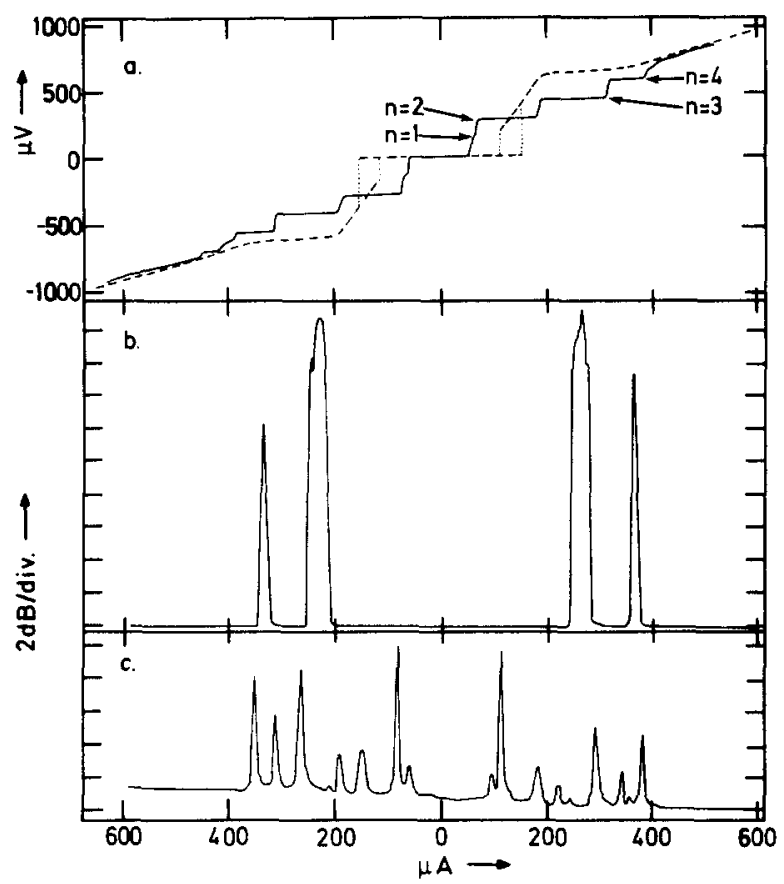

FIG. 2. (a) $I-V$ curve with (full) and without (dashed) 70-GHz microwaves (b) One-half harmonic spectrum (c) Power reflection coefficient at $35 \mathrm{GHz}$.

simply note that the response observed at a finite voltage bias does not differ significantly from the zero-voltage case.

Under different bias conditions the amplifier may start to oscillate at one-half the pump frequency, causing a severe reduction of the signal gain. This self-oscillation (one-half harmonic excitation) was observed on every rf-induced step of the order $n=1-7$. Consequently, the junction may act as a parametric device on all these steps. We have seen, in fact, on all steps $n \leqslant 7$, a response with qualitative features as in Fig. 1.

Preliminary experiments ${ }^{7}$ at $35 \mathrm{GHz}$ with a pump at 70 $\mathrm{GHz}$ have shown that the parametric effects persist in $\mathrm{Sn}-\mathrm{O}$ $\mathrm{Sn}$ tunnel junctions at these frequencies. We have observed self-oscillation at one-half the pump frequency at bias points on the rf-induced steps of the order $n=1-4$. Amplification with net gain has not yet been obtained. In Fig. 2 the amplitudes of the one-half harmonic excitation and of the reflected signal is shown versus dc-bias current. The two components were separated by $140 \mathrm{MHz}$ in this particular case and were detected in the upper and lower sidebands of the $\mathrm{Ka}$-band receiver. Hence, the idler $140 \mathrm{MHz}$ further off was not recorded. The parametric response at the fourth-order step corresponds to a voltage bias at $580 \mu \mathrm{V}$, which is approaching the energy gap $2 \Delta$ (at the temperature of this experiment $2 \Delta=650 \mu \mathrm{V}$ ).

Below we shall demonstrate that the parametric response on rf-induced steps may be accounted for in simple terms by an extension of the theory valid for the zero-voltage case. ${ }^{5}$ We assume that the junction is adequately described by the shunted junction model, neglecting the $\cos \phi \operatorname{conduc}$ tance and the frequency dependence of the quasiparticle resistance and the supercurrent.
On the step of order $n$, i.e., at the dc voltage $V_{n}=n h f_{p} / 2 e$ where $f_{p}$ is the pump frequency, we assume a junction voltage with a time dependence

$$
V(t)=V_{n}+\sum_{k} V_{k} \cos \left(2 \pi f_{k} t+\theta_{k}\right)
$$

where the summation includes components at the pump, signal, and idler frequencies ( $k=p, s$, and $i$, respectively). The amplitude of the pump component, $V_{p}$, may be large while the signal and idler components, $V_{s}$ and $V_{i}$, are small. Mixing products other than $V_{i}$ at $f_{i}=f_{p}-f_{s}$ are neglected in Eq. (1). In order to determine the amplitude and phase of the frequency components in Eq. (1) we insert the voltage $V(t)$ into the Josephson equations

$$
\frac{d \phi}{d t}=\frac{2 e V}{\hbar}, \quad I=I_{c} \sin \phi,
$$

and find the time-varying current carried by the ideal Josephson element

$$
I=I_{c} \sin \left(\phi_{0}+2 \pi n f_{p} t+\sum_{k} \phi_{k} \sin \left(2 \pi f_{k} t+\theta_{k}\right)\right),
$$

where $\phi_{k}=2 e V_{k} / h f_{k}$ and $k=p, s, i$. Expanding Eq. (3) in a Fourier series in the small-signal limit, $\phi_{s}, \phi_{i}<1$, and adding the currents through the quasiparticle resistance $R$ and the shunt capacitance $C$, the total current flowing in the junction is obtained. Equations relating the voltage to the input power at the various frequencies are then easily derived. The response is linear at the signal frequency and we may define an input admittance $Y_{s}=I_{s} / V_{s}$. If the impedance of the input/output waveguide is $Z_{0}$, the power gain at $f_{s}$ is

$$
\begin{aligned}
\Gamma_{s, n} & =\left|Z_{0} Y_{s}-1\right|^{2} /\left|Z_{0} Y_{s}+1\right|^{2} \\
& =\frac{\left[\eta^{2}\left(1-\sigma^{2}\right)+\eta^{2} A_{n}^{2}-B_{n}^{2}\right]^{2}+4 \eta^{4} \sigma^{2} A_{n}^{2}}{\left[\eta^{2}(1+\sigma)^{2}+\eta^{2} A_{n}^{2}-B_{n}^{2}\right]^{2}}
\end{aligned}
$$

where $\sigma=R / Z_{0}$ and $\eta=h f_{s} / 2 e R I_{c^{*}}$. The quantities $A_{n}$ and $B_{n}$ are given by

$$
\begin{aligned}
& \eta A_{n}=\left(f_{s} / f_{0}\right)^{2}-(-1)^{n} J_{n}\left(\phi_{p}\right) \cos \phi_{0}, \\
& B_{n}^{2}=\left[n J_{n}\left(\phi_{p}\right) \cos \phi_{0} / \phi_{p}\right]^{2}+\left[J_{n}^{\prime}\left(\phi_{p}\right) \sin \phi_{0}\right]^{2}
\end{aligned}
$$

where we have introduced the maximum plasma frequency $f_{0}=\left(2 e I_{c} / \hbar C\right)^{1 / 2} / 2 \pi . J_{n}\left(\phi_{p}\right)$ and $J_{n}^{\prime}\left(\phi_{p}\right)$ are the Bessel function of order $n$ and its derivative. The index $n$ of $\Gamma_{s, n}$ indicates bias on the $n$ th-order pump-induced step. The relation between the dc-bias current, $I_{\mathrm{dc}}$, and the dc phase $\phi_{0}$ is

$$
I_{\mathrm{dc}}=V_{n} / R+(-1)^{n} J_{n}\left(\phi_{p}\right) I_{c} \sin \phi_{0} .
$$

From Eq. (6) we see that within the model the $n$ th-order step is symmetric with respect to the Ohmic line $I=V_{n} / R$ with height $I_{c} J_{n}\left(\phi_{p}\right)$; this is in disagreement with the $I-V$ curves in Figs. 1 and 2. Nevertheless Eqs. (4)-(6) do reproduce the qualitative features of the experimental results.

Equation (4) has the same general structure as found for zero-voltage bias ${ }^{5}$ (included here as the special case $n=0$ ). The denominator consists of three terms:

(i) The term $\eta^{2}(1+\sigma)^{2}$ accounts for circuit losses. 
(ii) The term $\eta^{2} A_{n}^{2}$ is resonant if

$$
f_{s}^{2}=(-1)^{n} f_{0}^{2} J_{n}\left(\phi_{p}\right) \cos \phi_{0} \text {. }
$$

In the case $n=0$, the right-hand side is recognized as the plasma resonance. Equation (7) may hence be interpreted as a plasma resonance associated with the $n$th $\mathrm{rf}$ step.

(iii) The "parametric" term $B_{n}^{2}$, which may outbalance the circuit losses and the detuning from resonance, results in large or even infinite gain [Eq. (4)]. In the latter case, the amplifier becomes unstable and starts to oscillate. ${ }^{8}$

The simple theory outlined above does explain the observed parametric behavior of the Josephson junction biased on the rf-induced steps. We cannot, however, present a quantitative discussion until a detailed theory has been worked out which includes the frequency dependence of the pair current and the quasiparticle resistance. The noise properties of the device also deserves careful consideration.

The experiments indicate that it is feasible to realize parametric amplification at dc-bias points on every rf-induced step at least up to the energy gap.

Depending on the junction and the circuit parameters, we have often found it more favorable to select a bias point on one of the rf steps rather than on the supercurrent. We have, e.g., achieved gain at $\mathrm{rf}$-induced steps in a junction where no parametric effects were seen at the zero-voltage step $(n=0)$.

This work was supported in part by "Statens Naturvidenskabelige Forskningsråd" Grant No.: 811-8048.

'P.T. Parish and R.Y. Chiao, Appl. Phys. Lett. 25, 627 (1974).

${ }^{2} Y$. Taur and P.L. Richards, J. Appl. Phys. 48, 1321 (1977).

${ }^{3}$ S. Wahlsten, S. Rudner, and T. Claeson, Appl. Phys. Lett. 30, 298 (1977).

${ }^{4} J$. Mygind, N.F. Pedersen, and O.H. Soerensen, Appl. Phys. Lett. 32, 70 (1978).

'O.H. Soerensen, J. Mygind, and N.F. Pedersen, AIP Conf. Proc. 44, 246 (1978).

${ }^{6}$ R.Y. Chiao, M.J. Feldman, D.W. Peterson, B.A. Tucker, and M.T. Levinsen, see Ref. 5.

'The work at $70 / 35 \mathrm{GHz}$ was done in collaboration with M.T. Levinsen, The Oersted Institute, Univ. of Copenhagen.

${ }^{8}$ It may be shown by more careful evaluation that infinite gain may occur only at $f_{s}=\frac{1}{3} f_{p}$, explaining why this particular frequency is selected. 